\title{
CORRESPONDENCE
}

\section{Azithromycin in cystic fibrosis}

\section{To the Editors:}

We have read with interest the article by SOUTHERN and BARKER [1]. The authors critically reviewed the evidence from the randomised, controlled trials of the role of azithromycin in the management of cystic fibrosis (CF). In the three randomised, controlled studies described, the effects of up to 6 months of therapy with azithromycin in CF have been reported [2-4]. However, the controlled setting of randomised trials may differ from the clinical practice. Therefore, we can understand the authors' cautious closing remark on the precise role of azithromycin in the clinical setting.

We have recently audited our use of long-term azithromycin therapy in adult patients attending the Regional Adult CF unit, Leeds, UK [5]. A total of 36 patients (17 female), all of whom were colonised with Pseudomonas aeruginosa, had $500 \mathrm{mg}$ of azithromycin 3 times $^{-w}$ week $^{-1}$ for a mean \pm SD period of $9 \pm 6$ months. Mean age, Northern chest radiograph score, Shwachman-Kulczycki score and forced expiratory volume in one second (FEV1) were $24 \pm 7 \mathrm{yrs}, 12 \pm 3,68 \pm 14$, and $1.9 \pm 0.9 \mathrm{~L}$, respectively. In the group as a whole, there was no overall change in lung function during the treatment period. However, we were able to identify a subgroup of 24 patients in whom therapy with azithromycin was associated with a mean \pm SD increase in FEV1 by $0.3 \pm 0.2$ L. This group had significantly lower levels of antibodies to $P$. aeruginosa $(\mathrm{p}=0.007)$, with a median (range) of $19(4-136)$ versus $40(5-325)$ $\mathrm{U} \cdot \mathrm{mL}^{-1}$, and a lower number of exacerbations requiring i.v. antibiotics in the 12 months prior to starting azithromycin (3 (1-6) versus $5(2-7) ; \mathrm{p}=0.01)$ when compared to those patients where lung function remained unchanged.

We believe that our findings provide additional information on the clinical use of azithromycin. First, not all patients colonised with $P$. aeruginosa improve with azithromycin therapy. Secondly, the CF patients in whom treatment with azithromycin is more likely to be associated with improvements in lung function are characterised by a lower immunological response to $P$. aeruginosa and a lower frequency of exacerbations.

Our data suggest that therapy with azithromycin should be considered earlier in the disease process. Further data on whether therapy should be introduced before colonisation with Pseudomonas aeruginosa occurs is required.

\section{J.A. Kastelik, D.G. Peckham and S. Conway}

Adult Cystic Fibrosis Unit, Leeds Teaching Hospitals, Seacroft Hospital, Leeds, UK.

\section{REFERENCES}

1 Southern KW, Barker PM. Azithromycin in cystic fibrosis. Eur Respir J 2004; 24: 834-838.
2 Wolter J, Seeney S, Bell S, Bowler S, Masel P, McCormack J. Effect of long term treatment with azithromycin on disease parameters in cystic fibrosis: a randomised trial. Thorax 2002; 57: 212-216.

3 Equi A, Balfour-Lynn IM, Bush A, Rosenthal M. Long term azithromycin in children with cystic fibrosis: a randomised, placebo-controlled crossover trial. Lancet 2002; 360: 978-984.

4 Saiman L, Marshall BC, Mayer-Hamblett N, et al. Azithromycin in patients with cystic fibrosis chronically infected with Pseudomonas aeruginosa: a randomized controlled trial. JAMA 2003; 290: 1749-1756.

5 Kastelik JA, Patel T, Peckham DG, Etherington C, Conway SP. Macrolides in cystic fibrosis: clinical experience. Eur Respir J 2004; 24: Suppl. 48, A615s.

From the authors:

J.A. Kastelik and colleagues propose early use of azithromycin for cystic fibrosis (CF) based on subgroup analysis of 24 adult "responders" in their clinic who had lower Pseudomonas aeruginosa antibodies and less respiratory exacerbation prior to starting azithromycin compared to 12 nonresponders. Would these adult responders with mild respiratory disease have experienced a mean $0.3 \mathrm{~L}$ improvement in forced expiratory volume in one second with placebo? Subgroup post hoc analysis of retrospective data is fraught with bias and interpretation must be circumspect. The bottom line is we do not know why azithromycin improves respiratory function in people with CF and we do not know when to prescribe it. There is no clinical evidence, as yet, of an indirect antipseudomonal effect and, furthermore, the data of KASTELIK et al. [1] do not appear to support this assertion.

Is it possible that the widespread use of azithromycin in $\mathrm{CF}$ clinics throughout the world represents long-term antistaphyloccocal chemoprophylaxis being introduced via the back door? If so, we need to be cautious, as azithromycin has potential for generating resistance. This may have profound long-term effects on our choice of antimicrobials. There are probably better long-term anti-staphylococcal agents with a narrower spectrum of action and less tendency to promote resistance.

It is important that cystic fibrosis teams introduce azithromycin in a structured manner and audit their results, as J.A. Kastelik and colleagues have done. Close liaison with the microbiology department to monitor clinic resistance patterns is essential. 